\title{
Stability of fixed points of set-valued mappings and strategic stability of Nash equilibria
}

\author{
Shuwen Xiang ${ }^{\mathrm{a}}$, Shunyou Xia ${ }^{\mathrm{a}, \mathrm{b}, *}$, Jihao He ${ }^{\mathrm{a}}$, Yanlong Yang ${ }^{\mathrm{a}}$, Chenwei Liu ${ }^{\mathrm{a}}$ \\ a School of Mathematics and Statistics, Guizhou University, Guiyang, 550025, P. R. China. \\ ${ }^{b}$ School of Mathematics and Computer Science, Guizhou Education University, Guiyang, 550018, P. R. China. \\ Communicated by S. S. Chang
}

\begin{abstract}
In this paper, we mainly focus on the stability of Nash equilibria to any perturbation of strategy sets. A larger perturbation, strong $\delta$-perturbation, will be proposed for set-valued mapping. The class of perturbed games considered in the definition of strong $\delta$-perturbation is richer than those considered in many other definitions of stability of Nash equilibria. The strong $\delta$-perturbation of the best reply correspondence will be used to define an appropriate stable set for Nash equilibria, called SBR-stable set. As an SBR-stable set is stable to any strong $\delta$-perturbation and, various perturbations of strategy sets are not beyond the range of strong $\delta$-perturbation, it has the stability that various stable sets possess, such as fully stable set, stable set, quasistable set, and essential set. An SBR-stable set is stable to any perturbation of strategy sets, so it will provide convenience for study in strategic stability, which is even used to study any noncooperative game. (C)2017 All rights reserved.
\end{abstract}

Keywords: Stability, Nash equilibria, fixed point, strong $\delta$-perturbation, stable set.

2010 MSC: 90C29, 90C31, 91A10.

\section{Introduction}

The concept of equilibria, as defined by Nash (1951), is a core of the theory of noncooperative games. One reason for the widespread use of the Nash equilibria is that it has the advantage of existing in broad classes of games. As many games have multiple equilibria, a basic problem is the following: how can the players consistently predict the common equilibria? Many papers, some of which will be discussed here, have stressed that in some games, none of the Nash equilibria could be seen as a "reasonable" prediction. Hence the tough task for game theorists is not the existence of equilibria but the multiplicity.

Since Nash equilibria has multiplicity, the natural question that arises is: how to find a "reasonable" mechanism to refine equilibria? In addressing multiplicity, game theorists have examined a variety of arguments that refine the set of equilibria. Wu and Jiang [23] have proposed essential equilibria. Selten [19] has proposed trembling hand perfect equilibria. Myerson [15] and Kalai-Samet [9] have proposed proper and persistent equilibria. Kreps and Wilson [12] have proposed a variant-sequential equilibria.

\footnotetext{
${ }^{*}$ Corresponding author

Email addresses: shwxiang@vip.163.com (Shuwen Xiang), xiashunyou@126.com (Shunyou Xia), hejihao78@sohu.com (Jihao He), yylong1980@163.com (Yanlong Yang), liuchenwei15@163.com (Chenwei Liu)
}

doi:10.22436/jnsa.010.07.20 
The refinement of equilibria should follow certain rational principle, so a reasonable approach for refinement, as used in the above concepts is to select equilibria that are "stable" to slight perturbation in the specification of the game. An equilibrium is stable if each nearby game, obtained by perturbing each player's strategies by a "tremble", has a nearby equilibrium. Kohlberg and Mertens [11] introduced the concept of KM equilibria based on many refinement methods of Nash equilibria. One of the core ideas of KM equilibria is to seek the set of Nash equilibrium points that is stable to the slight perturbation of strategy sets. Kohlberg and Mertens established various stable sets, such as hyperstable set, fully stable set, and stable set. Unfortunately, none of these stable sets can satisfy all conditions of KM equilibria. Since then, many researchers including Mertens were committed to the improvement of stable set, and there has been a number of literatures researched on this field (see [4-8, 13, 14, 16, 20-22]). The most important concept of stable set is based on trembling hand perfect equilibria, which is stable to the perturbation of strategy sets. The stability is an important method for refining the equilibria, and the stability of these sorts of results in the literatures is usually dependent on the fact that the games were perturbed slightly in terms of the actions that players can take, the information that the players might have, or the underlying uncertainties about strategy sets.

It is more difficult to prove directly the connectedness of a stable set of Nash equilibria by game model itself. A usual approach is to apply the equivalence between equilibrium points and solutions of some nonlinear problems. Then, two ways can be followed. The first one consists in defining a stable set of Nash equilibria by using the equivalence between Nash equilibria and the fixed points of the best reply correspondence. The second one aims to translate Nash equilibrium points into solutions of Ky Fan minimax inequality. Yu and Xiang [26] proposed an essential set for Nash equilibria and proved the existence of essential component in the second direction by considering perturbations of payoff functions of players. There is a lot of researches about using the essential component to discuss the stability of Nash equilibria (see, $[1,2,8,17,18,24,25,27]$ ). However, there are two drawbacks in the second way. First, the minimax inequality related to the payoff function does not concern how players choose their strategies, and so the perturbation no longer has the real meaning of players' behavior. Second, it is difficult to consider the perturbation of strategy sets. Therefore, the first way was usually used to establish stable set to avoid these two drawbacks, for the reason that the best reply correspondence not only represented players' choice of strategy but also was used to define a stable set based on perturbations of strategy sets. Unfortunately, there is another, more pressing problem: to prove the existence of stable set by the best reply correspondence needs the continuity between best reply correspondences and strategy sets, but the perturbations of best reply correspondences are not continuous with respect to the perturbations of strategy sets when these perturbations are defined by Hausdorff distance simply. That is to say, when a perturbation of strategy set is sufficiently small, the perturbation of corresponding best reply correspondence does not need to be sufficiently small as well (see Example 3.2). To overcome this deficiency, Hillas [5] proposed a concept of quasistable set by using the perturbation of best reply correspondence instead of perturbation of strategy sets. The concept of quasistable set could avoid the discontinuity between two types of perturbations and satisfy the conditions of KM-equilibrium. But quasistable set may not be stable to perturbations of strategy sets, as the perturbation of best reply correspondence does not need to be sufficiently small, even the perturbation of strategy sets is small enough. Thus, some concepts of stable set, such as homotopy-stable set (see, Mertens [14]), essential set (see, McLennan [13]), CKM-set, and CT-set (see, Hillas et al. [6]), were presented, where a perturbation of strategy sets was defined with some additional conditions. Mclennan [13] introduced a related type of stable set called essential sets. A larger class of mappings was used to perturb the best reply correspondence and, more importantly, a coarser topology was used to define a perturbation.

A number of stability concepts were used to refine Nash equilibria. However, whether a stable set is stable to any perturbation of strategy sets is still a question. It is the reason why we consider a class of stronger perturbations for best reply correspondences to define an appropriate stable set. Since the stability of Nash equilibria to perturbations of strategy sets is a important problem in such research, a stable set will be proposed to ensure that it is stable to any perturbation defined by Hausdorff distance 
between strategy sets simply. As mentioned above, when a perturbation of strategy sets is sufficiently small, the perturbation of corresponding best reply correspondence is not necessarily sufficiently small. Hence the key to the definition of stable set is to find a reasonable class of perturbations for best reply correspondence and to answer the following two problems at the same time.

1. When the perturbation of a strategy set is sufficiently small, is the corresponding perturbation of the best reply sufficiently small as well?

2. Does the stable set defined by these perturbations still has existence and connectedness?

Around these two problems, a larger perturbation, strong $\delta$-perturbation, will be proposed for setvalued mappings. It will be proved that when a perturbation of strategy sets is sufficiently small, the strong $\delta$-perturbation of the best reply correspondence is sufficiently small as well. The class of perturbed games considered in the definition of strong $\delta$-perturbation is richer than those considered in many other definitions of stability of Nash equilibria. The strong $\delta$-perturbation of the best reply correspondence will be used to define an appropriate stable set for Nash equilibria, called SBR-stable set. An SBR-stable set is stable to any strong $\delta$-perturbation, and various form of perturbations of strategy sets are not beyond the range of strong $\delta$-perturbation, so an SBR-stable set has the stability that various stable sets possess, such as, fully stable set, stable set, quasistable set and essential set. An SBR-stable set is defined for any compact convex subset in normed space and the perturbation of strategy sets is defined directly by distance between sets without any additional condition, so the concept of SBR-stable set will provide convenience for the study in strategic stability, which is even used to study any noncooperative game.

\section{Preliminaries}

For convenience, we recall some definitions and conclusions.

Definition 2.1. Let $Y$ be a Hausdorff topological space, and $\left\{A_{\alpha}\right\}$ be a net constituted by the subsets of $Y$.

(1) $x$ is said to be a limit point of $\left\{A_{\alpha}\right\}$, if for any neighborhood $U$ of $x$, there is $\alpha_{0}$ such that $A_{\alpha} \cap U \neq \emptyset$ for all $\alpha \geqslant \alpha_{0}$. The set of all limit points of $\left\{A_{\alpha}\right\}$ is denoted as $\lim \inf A_{\alpha}$.

(2) $x$ is said to be a cluster point of $\left\{A_{\alpha}\right\}$, if for any neighborhood $U$ of $x$, and any $\alpha_{0}$, there is some $\alpha \geqslant \alpha_{0}$ such that $A_{\alpha} \cap U \neq \emptyset$. The set of all cluster points of $\left\{A_{\alpha}\right\}$ is denoted as $\lim \sup A_{\alpha}$.

(3) If $\liminf A_{\alpha}=\limsup A_{\alpha}=A$, then $A_{\alpha}$ converges to $A$, denoted by $\lim A_{\alpha}=A$.

The following lemma is derived from Theorem 3.3.11 and Corollary 4.2.3 of Klein-Thompson [10].

Lemma 2.2. Suppose that $Y$ is a compact metric space and that $\left\{A_{\alpha}\right\}$ is a net comprised of nonempty closed subsets of $\mathrm{Y}$. Then $\lim A_{\alpha}=A$ if and only if $A_{\alpha}$ converges to $A$ with respect to Hausdorff metric.

Unless otherwise stated, $X$ is supposed to be a nonempty compact and convex subset in the normed space $E . B_{\varepsilon}(x)$ is used to represent $\varepsilon$-neighbourhood of $x$ no matter which space $x$ is in.

Let

$\mathcal{C}(X)=\left\{T \mid T: X \mapsto 2^{X}\right.$ is an upper semi-continuous mapping with nonempty compact and convex value $\}$.

For any $T \in \mathcal{C}(X)$, fix $(T)$ is used to represent the set of all the fixed points of $T$. By Fan-Glicksberg fixed-point theorem, fix $(T)$ is non-empty and compact.

As usual, the distance between set-valued mappings $T_{1}, T_{2} \in \mathcal{C}(X)$ is defined by

$$
\rho_{C}\left(T_{1}, T_{2}\right)=\sup _{x \in X} H_{d}\left(T_{1}(x), T_{2}(x)\right),
$$

where $\mathrm{H}_{\mathrm{d}}$ is the Hausdorff metric between nonempty closed subsets of $\mathrm{X}$.

The concept of essential fixed point is first proposed by Fort [3]. Then the method was widely used to the stability of solutions of optimization and game theory. 
Definition 2.3. Let $T \in \mathcal{C}(X)$. A $\delta$-neighborhood of $T$ is defined by

$$
P(T, \delta)=\left\{T^{\prime} \in \mathcal{C}(X): \rho_{C}\left(T^{\prime}, T\right)<\delta\right\} .
$$

Definition 2.4. Let $T \in \mathcal{C}(X)$, and $e(T) \subset \operatorname{fix}(T)$ be a nonempty closed subset. For any $\varepsilon>0$, if there is a $\delta>0$ such that $\left(e(T)+B_{\varepsilon}(0)\right) \cap \operatorname{fix}\left(T^{\prime}\right) \neq \emptyset$ for all $T^{\prime} \in P(T, \delta)$, then $e(T)$ is called an essential fixed point set with respect to $\left(\mathcal{C}(X), \rho_{C}\right)$, or an essential set for short.

\section{Remark 2.5.}

(1) If an essential set $e(T)$ is a single point set, i.e., $e(T)=\left\{x^{*}\right\}$, then $x^{*}$ is an essential fixed point, or essential point in short.

(2) $e_{1}(T), e_{2}(T)$ are two nonempty closed subsets of $X$ and $e_{1}(T) \subset e_{2}(T)$. If $e_{1}(T)$ is essential, then $e_{2}(T)$ is essential as well.

We introduce some basic definitions.

An n-person noncooperative game is denoted as follows:

1. $\mathbf{N}=\{1,2, \cdots, \mathfrak{n}\}$ is a finite set of players;

2. $X_{i}$ is the strategy set of player $i$, and $X=\prod_{i=1}^{n} X_{i}$ is the strategy profile space. For any $i \in N$, denote $X_{-i}=\left(X_{1}, \cdots, X_{i-1}, X_{i+1}, \cdots, X_{n}\right)$ and $x_{-i}=\left(x_{1}, \cdots, x_{i-1}, x_{i+1}, \cdots, x_{n}\right) \in X_{-i} ;$

3. $f=\left(f_{1}, \cdots, f_{n}\right): X \mapsto \mathbb{R}^{n}$ is the payoff, where $f_{i}: X=\prod_{i=1}^{n} X_{i} \mapsto \mathbb{R}$ is the payoff function of player i. $\Gamma=\left(N ;\left(X_{1}, X_{2}, \cdots, X_{n}\right) ;\left(f_{1}, f_{2}, \cdots, f_{n}\right)\right)$ is called an n-person noncooperative game, denoted by $\Gamma(X, f)=\left(N ;\left(X_{i}\right) ;\left(f_{i}\right)\right)$.

$x^{*}=\left(x_{i}^{*}, x_{-i}^{*}\right)$ is said to be a Nash equilibrium point of $\Gamma(X, f)$, if

$$
f_{i}\left(x_{i}^{*}, x_{-i}^{*}\right)=\max _{y_{i} \in X} f_{i}\left(y_{i}, x_{-i}^{*}\right), \quad \forall i \in N .
$$

Let

$$
\Gamma=\{\Gamma(X, f): \Gamma(X, f) \text { satisfies all conditions of Theorem 2.6 }\}
$$

As to the existence of Nash equilibria, Nikado and Isodo proved the following conclusion.

Theorem 2.6. Let $\Gamma(X, f)$ be an n-person noncooperative game and satisfy the following conditions:

(1) for each $i \in N, X_{i}$ is a nonempty compact convex subset in the normed space $E_{i}$;

(2) for each $i \in N$, $f$ is continuous on $X$;

(3) for each $i \in N$ and any fixed $x_{-i} \in X_{-i}, f_{i}\left(x_{i}, x_{-i}\right)$ is quasi-concave on $X_{i}$.

Then $\Gamma(X, f)$ has a Nash equilibrium point in $X$, and the set of all the Nash equilibrium points of $\Gamma(X, f)$ is denoted by $\mathrm{E}(\mathrm{X}, \mathrm{f})$.

For any $\Gamma(X, f) \in \Gamma$, its best reply correspondence is defined as follows:

(i) for each $i \in N$, the best reply correspondence of player $i$ is given by

$$
\operatorname{BR}_{(X, f)}^{i}\left(x_{-i}\right)=\left\{x_{i} \in X_{i}: f_{i}\left(x_{i}, x_{-i}\right) \geqslant f_{i}\left(y_{i}, x_{-i}\right), \forall y_{i} \in X_{i}\right\}, \quad \forall x_{-i} \in X_{-i} ;
$$

(ii) the best reply correspondence $B R_{(X, f)}: X \mapsto 2^{X}$ of $\Gamma(X, f)$ is given by

$$
\mathrm{BR}_{(X, f)}\left(x_{i}, x_{-i}\right)=\prod_{i \in N} B_{(X, f)}^{i}\left(x_{-i}\right), \quad \forall x=\left(x_{i}, x_{-i}\right) \in X .
$$

The set of fixed points of mapping $B R_{(X, f)}$ is denoted by $\operatorname{fix}\left(B R_{(X, f)}\right)$. By definition of Nash equilibria, $x^{*} \in E(X, f)$ if and only if $x^{*} \in \operatorname{fix}\left(B R_{(X, f)}\right)$. Then it is easy to see from the proof of the existence of 
Nash equilibria that the best reply correspondence $B R_{(X, f)}$ is an upper semi-continuous mapping with nonempty compact and convex value on $\mathrm{X}$, namely, $\mathrm{BR}_{(\mathrm{X}, \mathrm{f})} \in \mathcal{C}(\mathrm{X})$.

\section{Strong $\delta$-perturbation and strongly essential set of fixed points}

An n-person noncooperative finite game is denoted by $\Gamma(S, p)=\left(N ;\left(S_{i}\right) ;\left(p_{i}\right)\right)$ where strategy set $S_{i}=\left\{s_{1}^{i}, s_{2}^{i}, \cdots, s_{m i}^{i}\right\}$ is a finite set. Let us consider its mixed strategy model where mixed strategy sets and payoff functions are defined as follows:

$$
\begin{aligned}
\Delta_{i} & =\left\{x_{i}=\left(u_{1}^{i}, \cdots, u_{m i}^{i}\right): 0 \leqslant u_{j i}^{i} \leqslant 1, j i=1, \cdots, m i, \sum_{j i=1}^{m i} u_{j i}^{i}=1\right\}, \Delta=\prod_{i \in N} \Delta_{i}, \\
f_{i}\left(x_{1}, x_{2}, \cdots, x_{n}\right) & =\sum_{j 1=1}^{m 1} \sum_{j 2=1}^{m 2} \cdots \sum_{j n=1}^{m n} u_{j 1}^{1} u_{j 2}^{2} \cdot u_{j n}^{n} p_{i}\left(s_{j 1}^{1}, s_{j 2}^{2}, \cdots, s_{j n}^{n}\right) .
\end{aligned}
$$

Denote by $\Gamma(X, f)=\left(N ;\left(\Delta_{i}\right) ;\left(p_{i}\right)\right)$ the mixed strategy form of $\Gamma(S, p)=\left(N ;\left(S_{i}\right) ;\left(p_{i}\right)\right)$.

For game $\Gamma(X, f)$, following Kohlberg and Mertens [11] and Selten [19], we will say that, an n-tuple $\widetilde{\epsilon}=\left(\widetilde{\epsilon}_{1}, \cdots, \widetilde{\epsilon}_{n}\right)\left(\widetilde{\epsilon}_{i}=\left(\epsilon_{1}^{i}, \cdots, \epsilon_{m i}^{i}\right), \epsilon_{j i}^{i}>0, j i=1, \cdots, m i, i \in N\right)$ gives rise to an $\epsilon$-perturbation of $\Gamma(X, f)=\left(N ;\left(\Delta_{i}\right) ;\left(p_{i}\right)\right)$ as

$$
\Delta^{\widetilde{\epsilon}}=\prod_{i \in N} \Delta_{i}^{\widetilde{\epsilon}}, \text { where } \Delta_{i}^{\widetilde{\epsilon}}=\left\{x_{i}=\left(u_{1}^{i}, \cdots, u_{m i}^{i}\right) \in \Delta_{i}: u_{j i}^{i} \geqslant \epsilon_{j i}^{i}, j i=1, \cdots, m i\right\} .
$$

Now let us consider the general form of the perturbation of strategy sets. Considering the general $n-$ person games, we propose the concept in a normed space.

Denote by $\mathcal{K}\left(X_{i}\right)$ the family of all non-empty compact and convex subsets of $X_{i}$. Let $X^{\prime}=\prod_{i \in N} X_{i}^{\prime}$, where $X_{i}^{\prime} \in \mathcal{K}\left(X_{i}\right)$, for all $i \in N$. $X^{\prime}$ gives rise to a perturbation game $\Gamma\left(X \mid X^{\prime}, f\right)$, whose strategy profile space is $X^{\prime}$. The payoff function of player $i$ is simply the restriction of $f_{i}$ to $X_{1} \times \cdots \times X_{i}^{\prime} \times \cdots X_{n}$, and the best reply correspondence is as follows:

$$
\mathrm{BR}_{\left(X^{\prime}, \mathfrak{f}\right)}\left(x_{i}, x_{-i}\right)=\prod_{i \in N} \operatorname{BR}_{\left(X^{\prime}, f\right)}^{i}\left(x_{-i}\right), \quad \forall x=\left(x_{i}, x_{-i}\right) \in X,
$$

where

$$
\operatorname{BR}_{\left(X^{\prime}, f\right)}^{i}\left(x_{-i}\right)=\left\{x_{i}^{\prime} \in X_{i}^{\prime}: f_{i}\left(x_{i}^{\prime}, x_{-i}\right) \geqslant f_{i}\left(y_{i}, x_{-i}\right), \forall y_{i} \in X_{i}^{\prime}\right\}, \quad \forall x_{-i} \in X_{-i} .
$$

For each game $\Gamma(X, f) \in \Gamma$, let

$$
\Lambda(\Gamma(X, f))=\left\{\Gamma\left(X \mid X^{\prime}, f\right): X^{\prime}=\prod_{i \in N} X_{i}^{\prime}, X_{i}^{\prime} \in \mathcal{K}\left(X_{i}\right), i \in N\right\} .
$$

Consider the perturbation of strategy sets of $\Gamma(X, f)$.

Definition 3.1. Let $\Gamma(X, f) \in \Gamma$. A $\delta$-neighborhood of $\Gamma(X, f)$ is defined by

$$
P(\Gamma(X, f), \delta)=\left\{\Gamma\left(X \mid X^{\prime}, f\right): X^{\prime}=\prod_{i \in N} X_{i}^{\prime}, X_{i}^{\prime} \in \mathcal{K}\left(X_{i}\right) \text { and } H_{d}\left(X^{\prime}, X\right)<\delta\right\} .
$$

Firstly, an example is given to illustrate that, the perturbation of the best reply correspondence with respect to $\rho_{C}$ does not need to be sufficiently small when the perturbation of strategy sets is sufficiently small.

Example 3.2. A two-person noncooperative game with payoffs is given in Table 1 . 
Table 1: Table of payoffs for Example 3.2.

\begin{tabular}{|c|c|c|c|c|}
\hline \multirow{4}{*}{ Player 1 } & \multicolumn{4}{|c|}{ Player 2 } \\
\cline { 2 - 5 } & & $\mathrm{e}$ & $\mathrm{F}$ & $\mathrm{g}$ \\
\cline { 2 - 5 } & $\mathrm{a}$ & $(1,0)$ & $(-1,1)$ & $(1,-1)$ \\
\cline { 2 - 5 } & $\mathrm{b}$ & $(1,0)$ & $(0,0)$ & $(-1,1)$ \\
\cline { 2 - 5 } & $\mathrm{c}$ & $(-1,1)$ & $(1,-1)$ & $(0,1)$ \\
\hline
\end{tabular}

The mixed strategy form of this game is denoted by $\Gamma(\Delta, u)$, where the mixed strategy sets of player 1 and player 2 are respectively as follows:

$$
\begin{aligned}
& \Delta_{1}=\left\{x_{1}=\left(u^{1}, u^{2}, u^{3}\right) \mid 0 \leqslant u^{i} \leqslant 1, \sum_{i=1}^{3} u^{i}=1\right\}=\operatorname{co}\{(1,0,0),(0,1,0),(0,0,1)\} \\
& \Delta_{2}=\left\{x_{2}=\left(v^{1}, v^{2}, v^{3}\right) \mid 0 \leqslant v^{i} \leqslant 1, \sum_{i=1}^{3} v^{i}=1\right\}=\operatorname{co}\{(1,0,0),(0,1,0),(0,0,1)\}
\end{aligned}
$$

Then the set of strategy profiles is $\Delta=\Delta_{1} \times \Delta_{2}$. For any $\delta>0$, the following perturbation of strategy set is considered.

$$
\begin{aligned}
\Delta_{1}^{\delta} & =\operatorname{co}\left\{(1,0,0),\left(\frac{\delta}{2}, 1-\delta, \frac{\delta}{2}\right),(0,0,1)\right\} \\
& =\left\{x_{1} \in X_{1} \mid x_{1}=\left(t^{1}+\frac{\delta}{2} t^{2},(1-\delta) t^{2}, \frac{\delta}{2} t^{2}+t^{3}\right), 0 \leqslant t^{i} \leqslant 1, \sum_{i=1}^{3} t^{i}=1\right\}, \\
\Delta_{2}^{\delta} & =\Delta_{2}, \\
\Delta^{\delta} & =\Delta_{1}^{\delta} \times \Delta_{2}^{\delta} .
\end{aligned}
$$

Then $\Gamma\left(\Delta \mid \Delta^{\delta}, \mathrm{u}\right) \in \Lambda(\Gamma(\Delta, \mathrm{u}))$. Denote the best reply correspondences of $\Gamma\left(\Delta \mid \Delta^{\delta}, \mathrm{u}\right)$ and $\Gamma(\Delta, \mathrm{u})$ by $B R_{\Delta}=$ $\mathrm{BR}_{\Delta}^{1} \times \mathrm{BR}_{\Delta}^{2}$ and $\mathrm{BR}_{\Delta^{\delta}}=\mathrm{BR}_{\Delta^{\delta}}^{1} \times \mathrm{BR}_{\Delta^{\delta}}^{2}$, respectively, where

$$
\mathrm{BR}_{\Delta}^{1}((1,0,0))=\left\{x_{1}=\left(\mathfrak{u}^{1}, \mathrm{u}^{2}, 0\right) \mid 0 \leqslant u^{1} \leqslant 1,0 \leqslant u^{2} \leqslant 1, u^{1}+u^{2}=1\right\}, \quad B R_{\Delta^{\delta}}^{1}((1,0,0))=\{(1,0,0)\} .
$$

Hence,

$$
\begin{aligned}
\Gamma\left(\Delta \mid \Delta^{\delta}, \mathrm{u}\right) & \in \mathrm{P}(\Gamma(\Delta, \mathrm{u}), \delta), \\
\rho_{\mathrm{C}}\left(\mathrm{BR}_{\Delta}, \mathrm{BR}_{\Delta^{\delta}}\right) & =\sup _{x \in \Delta} \mathrm{H}_{\mathrm{d}}\left(\mathrm{BR}_{\Delta}(\mathrm{x}), \mathrm{BR}_{\Delta^{\delta}}(\mathrm{x})\right) \\
& \geqslant \mathrm{H}_{\mathrm{d}}\left(\mathrm{BR}_{\Delta}((1,0,0)), \mathrm{BR}_{\Delta^{\delta}}((1,0,0)) \geqslant \mathrm{H}_{\mathrm{d}}\left(\mathrm{BR}_{\Delta}^{1}((1,0,0)), \mathrm{BR}_{\Delta^{\delta}}^{1}((1,0,0))=\sqrt{2} .\right.\right.
\end{aligned}
$$

Notice that $\delta$ is arbitrary. It is clear that the perturbation of the best reply correspondence is not sufficiently small even the perturbation of strategy sets is small enough.

By Example 3.2, it is necessary to introduce an appropriate perturbation for a best reply correspondence to ensure that the perturbation can be sufficiently small whenever the perturbation of strategy sets is sufficiently small.

In addition, the following example is used to illustrate that it is necessary to improve the concept of essential fixed point for further refinement of fixed point set.

Example 3.3. Suppose a set-valued mapping $T:[0,1] \mapsto 2^{[0,1]}$ is

$$
T(x)= \begin{cases}(x-1)^{2}, & x \in\left[0, \frac{1}{2}\right] \cup\left[\frac{3}{4}, 1\right], \\ {\left[(x-1)^{2}, 1\right],} & x \in\left(\frac{1}{2}, \frac{3}{4}\right) .\end{cases}
$$

The graph of $\mathrm{T}$ is shown as Figure 1. 


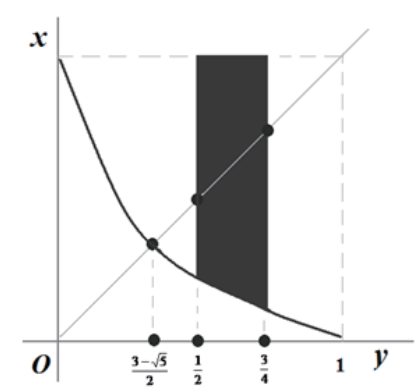

Figure 1: Essential and strongly essential.

The set of fixed points of $T$ is fix $(T)=\left\{\frac{3-\sqrt{5}}{2}\right\} \cup\left[\frac{1}{2}, \frac{3}{4}\right]$. By Definition 2.3, $\left\{\frac{3-\sqrt{5}}{2}\right\}$ and all the points in $\left[\frac{1}{2}, \frac{3}{4}\right]$ are essential fixed points of $\mathrm{T}$. Notice that there is a large difference between essential fixed point $\left\{\frac{3-\sqrt{5}}{2}\right\}$ and any point in $\left[\frac{1}{2}, \frac{3}{4}\right]$. By contrast, $\left\{\frac{3-\sqrt{5}}{2}\right\}$ has stronger stability and all the points in $\left[\frac{1}{2}, \frac{3}{4}\right]$ seem a little abnormal. It is the reason why we rewrite the condition of essential fixed point in a stronger perturbation. A perturbation that we think will help us well to find some fixed points more stable, called strongly essential fixed point. The concept of strongly essential fixed point should be introduced and all the points of $\left[\frac{1}{2}, \frac{3}{4}\right]$ will be eliminated.

In addition, it was imperative that the concept of stronger perturbation must ensure that every stable set (minimal essential set) is connected. Now, we define a strong perturbation as follows.

Definition 3.4. Let $T \in \mathcal{C}(X)$. A strong $\delta$-neighborhood of $T$ is defined by

$$
\mathrm{SP}(\mathrm{T}, \delta)=\left\{\mathrm{S} \in \mathcal{C}: \mathrm{S}(\mathrm{x}) \subset \operatorname{co}\left(\mathrm{T}\left(\mathrm{x}+\mathrm{B}_{\delta}(0)\right)+\mathrm{B}_{\delta}(0)\right), \forall x \in X\right\} .
$$

Remark 3.5. Definitions 2.3 and 3.4 imply $\mathrm{P}(\mathrm{T}, \delta) \subset \mathrm{SP}(\mathrm{T}, \delta)$, and then $\mathrm{SP}(\mathrm{T}, \delta)$ has a wider perturbation range than $\mathrm{P}(\mathrm{T}, \delta)$.

For any set-valued mapping $T, \operatorname{Gr}(T)$ denotes the graph of $T$. Let $\operatorname{GP}(T, \delta)=\{S \in \mathcal{C}(X): \operatorname{Gr}(S) \subset$ $\left.\mathrm{Gr}(\mathrm{T})+\mathrm{B}_{\delta}(0)\right\}$. Then $\mathrm{GP}(\mathrm{T}, \delta)$ denotes the graph perturbation of $\mathrm{T}$ introduced by McLennan [13]. It will be proved that $S P(T, \delta)$ has a wider perturbation range than $G P(T, \delta)$.

Lemma 3.6. If $\mathrm{T}^{\prime} \in \mathrm{GP}(\mathrm{T}, \delta)$, then $\mathrm{T}^{\prime}(\mathrm{x}) \subset \mathrm{T}\left(\mathrm{x}+\mathrm{B}_{\delta}(0)\right)+\mathrm{B}_{\delta}(0)$ for each $\mathrm{x} \in \mathrm{X}$, and hence $\mathrm{T}^{\prime} \in \mathrm{SP}(\mathrm{T}, \delta)$.

Proof. Let $\mathrm{T}^{\prime} \in \mathrm{GP}(\mathrm{T}, \delta)$. Then $\operatorname{Gr}\left(\mathrm{T}^{\prime}\right) \subset \mathrm{Gr}(\mathrm{T})+\mathrm{B}_{\delta}(0)$. It is immediate from $\operatorname{Gr}\left(\mathrm{T}^{\prime}\right) \subset \mathrm{Gr}(\mathrm{T})+\mathrm{B}_{\delta}(0)$ that

$$
\mathrm{T}^{\prime}(\mathrm{x}) \subset \mathrm{T}\left(\mathrm{x}+\mathrm{B}_{\delta}(0)\right)+\mathrm{B}_{\delta}(0), \quad \forall x \in \mathrm{X} .
$$

In fact, because, for each $x \in X$ and $y \in T^{\prime}(x)$, there are some $x^{\prime} \in X$ and $y^{\prime} \in T\left(x^{\prime}\right)$ such that $\left\|x-x^{\prime}\right\|<\delta$ and $\left\|y-y^{\prime}\right\|<\delta$. Then $y \in y^{\prime}+B_{\delta}(0) \subset T^{\prime}\left(x^{\prime}\right)+B_{\delta}(0) \subset T^{\prime}\left(x+B_{\delta}(0)\right)+B_{\delta}(0)$, and hence (3.1) holds. It is obvious that $T^{\prime} \in S P(T, \delta)$.

Definition 3.7. Let $T \in \mathcal{C}(X)$, and $e(T) \subset \operatorname{fix}(T)$ be a closed set. For any $\varepsilon>0$, if there is $\delta>0$ such that $\left(e(T)+B_{\varepsilon}(0)\right) \cap \operatorname{fix}\left(T^{\prime}\right) \neq \emptyset$ for all $T^{\prime} \in S P(T, \delta)$, then $e(T)$ is said to be a strongly essential set of fixed points with respect to the strong $\delta$-perturbation, or a strongly essential set for short. Moreover, if $e(T)$ is the minimal element of the family of all the strongly essential sets of fix $(T)$ ordered by the inclusion relation, then $e(T)$ is said to be a minimal essential set of fix $(T)$ and a connected minimal essential set is said to be a stable set.

\section{Remark 3.8.}

(1) If a strongly essential set $e(T)$ is a single point set, i.e. $e(T)=\left\{x^{*}\right\}$, then $x^{*}$ is a strongly essential fixed point, a strongly essential point for short. 
(2) $e_{1}(T), e_{2}(T)$ are two nonempty closed subsets of $X$ and $e_{1}(T) \subset e_{2}(T)$. If $e_{1}(T)$ is strongly essential, then $e_{2}(T)$ is strongly essential as well.

(3) If some component $c(T)$ of $\operatorname{fix}(T)$ is a strongly essential set, then $c(T)$ is said to be a strong essential connected component of fix $(\mathrm{T})$.

(4) By Remark 3.5, Definition 2.3, and Definition 3.7, a strongly essential set is clear a essential set. By Lemma 3.6, a strongly essential set is stable to BR-perturbations defined by Hausdorff distance between mappings or graph perturbations introduced by McLennan [13]. The reason for considering $\mathrm{SP}(T, \delta)$ rather than $\mathrm{GP}(T, \delta)$ as a strong perturbation is not only to give a stronger stability but also to ensure the connectedness of stable set.

To know the advantage of strongly essential set, consider the fixed point set fix $(T)$ in Example 3.3. By definition of strongly essential set, $\left\{\frac{3-\sqrt{5}}{2}\right\}$ is strongly essential, and $\left[\frac{1}{2}, \frac{3}{4}\right]$ is not. In fact, choose a single-valued mapping $T^{\prime}(x)=(x-1)^{2}, x \in[0,1]$, and then $T^{\prime} \in \operatorname{SP}(T, \delta)$ for all $\delta>0$. Notice that $\left(\left[\frac{1}{2}, \frac{3}{4}\right]+B_{\frac{1}{8}}(0)\right) \cap \operatorname{fix}\left(T^{\prime}\right) \subset\left(\frac{3}{8}, 1\right) \cap\left\{\frac{3-\sqrt{5}}{2}\right\}=\emptyset$. Hence, $\left[\frac{1}{2}, \frac{3}{4}\right]$ is not a strongly essential set and the set $\left[\frac{1}{2}, \frac{3}{4}\right]$ has been eliminated. Example 3.3 shows that if our goal is to discriminate between some essential fixed points and others, the concept of strongly essential set must be strengthened in some way.

The existence and connectedness of stable set of fixed points are proved as follows.

Theorem 3.9. Let $\mathrm{T} \in \mathcal{C}(\mathrm{X})$. Then fix $(\mathrm{T})$ has at least one minimal essential set.

Proof. Suppose $T \in \mathcal{C}(X)$. It is easy to prove that $\operatorname{fix}(T)$ is a closed set. A discussion of what followed fix $(T)$ is proved to be a strongly essential set of itself. It is sufficient to prove that for any $\varepsilon>0$, if there is $\delta>0$, such that $\left(\operatorname{fix}(T)+\mathrm{B}_{\epsilon}(0)\right) \cap \operatorname{fix}\left(T^{\prime}\right) \neq \emptyset$ for all $T^{\prime} \in \operatorname{SP}(T, \delta)$. Otherwise, there is some $\varepsilon_{0}>0$ and a sequence of positive numbers $\delta_{1}>\delta_{2}>\cdots>\delta_{n}>\cdots$ with $\delta_{n} \rightarrow 0$ and $T_{n} \in \operatorname{SP}(T, \delta)$, such that $\left(\operatorname{fix}(T)+B_{\epsilon_{0}}(0)\right) \cap \operatorname{fix}\left(T_{n}\right)=\emptyset$. Choose an arbitrary sequence $\left\{x_{n}^{*}\right\}$ such that $x_{n}^{*} \in \operatorname{fix}\left(T_{n}\right) .\left\{x_{n}^{*}\right\} \subset X$ and the compactness of $X$ imply that $\left\{x_{n}^{*}\right\}$ has a convergent subsequences. Without loss of generality, we may assume $x_{n}^{*} \rightarrow x_{0} . x_{n}^{*} \in \operatorname{fix}\left(T_{n}\right)$ and $T_{n} \in S P\left(T, \delta_{n}\right)$ imply $x_{n}^{*} \in T_{n}\left(x_{n}^{*}\right) \subset \operatorname{co}\left(T\left(x_{n}^{*}+B_{\delta_{n}}(0)\right)+B_{\delta_{n}}(0)\right)$. Since $T$ is upper semi-continuous, there exists $0<\eta<\varepsilon$, such that $T\left(B_{\eta}\left(x_{0}\right)\right) \subset T\left(x_{0}\right)+B_{\varepsilon}(0)$, and since $\delta_{n} \rightarrow 0$ and $x_{n} \rightarrow x_{0}$, there exists $N_{0}>0$ such that $\delta_{n}<\varepsilon, \delta_{n}<\frac{\eta}{2}$ and $x_{n}^{*} \in B_{\frac{\eta}{2}}\left(x_{0}\right)$ for all $n>N_{0}$, and therefore $\mathrm{B}_{\delta_{\mathfrak{n}}}\left(\mathrm{x}_{\mathrm{n}}^{*}\right) \subset \mathrm{B}_{\eta}\left(\mathrm{x}_{0}\right)$. Consequently,

$$
\begin{aligned}
x_{n}^{*} \in \operatorname{co}\left(T\left(x_{n}^{*}+B_{\delta_{\mathfrak{n}}}(0)\right)+B_{\delta_{\mathfrak{n}}}(0)\right) & \subset \operatorname{co}\left(T\left(B_{\mathfrak{\eta}}\left(x_{0}\right)\right)+B_{\varepsilon}(0)\right) \\
& \subset \operatorname{co}\left(T\left(x_{0}\right)+B_{\varepsilon}(0)+B_{\varepsilon}(0)\right)=\operatorname{co}\left(T\left(x_{0}\right)+B_{2 \varepsilon}(0)\right) .
\end{aligned}
$$

The convexity of $T\left(x_{0}\right)$ and $B_{2 \varepsilon}(0)$ imply $x_{n}^{*} \in T\left(x_{0}\right)+B_{2 \varepsilon}(0)$, and then $x_{0} \in T\left(x_{0}\right)+B_{3 \varepsilon}(0)$. Since $\varepsilon$ is arbitrary, it is obvious that $x_{0} \in T\left(x_{0}\right)$ and $x_{0} \in \operatorname{fix}(T)$. Moreover, $x_{n}^{*} \rightarrow x_{0}$ implies $x_{n}^{*} \in x_{0}+B_{\varepsilon_{0}}(0)$, and then $x_{n}^{*} \in\left(\operatorname{fix}(T)+B_{\varepsilon_{0}}(0)\right) \cap \operatorname{fix}\left(T_{n}\right) \neq \emptyset$ for sufficiently large $n$, a contradiction, and hence fix $(T)$ is a strongly essential set of itself. Let $\mathfrak{R}$ denote the family of all the strongly essential sets of fix $(T)$. Then $\Re \neq \emptyset$.

For any decreasing chain in $\mathfrak{R}$, it is sufficient to show there is a lower bound for $\mathfrak{R}$ and we may apply Zorn lemma to get the minimal element of $\Re$.

Suppose that $\left\{e_{\alpha}\right\}$ is a decreasing chain and that $D$ is a index set, i.e. $e_{\alpha_{1}} \subset e_{\alpha_{2}}$ when $\alpha_{1} \geqslant \alpha_{2}$. It follows from the compactness of $e_{\alpha}$ and finite intersection property that $e=\bigcap_{\alpha \in D} e_{\alpha}$ is a nonempty compact set. Now we prove $\lim e_{\alpha}=e$. Since $\left\{e_{\alpha}\right\}$ is a decreasing chain and $e=\bigcap_{\alpha \in D}^{\alpha \in D} e_{\alpha}, \limsup e_{\alpha}=\liminf e_{\alpha}$ and $e \subset \limsup e_{\alpha}$. It remains to show $\lim \sup e_{\alpha} \subset e$. If not, then there exists $x \in \lim \sup e_{\alpha}$ such that $x \notin e$, and then there is an $\alpha_{0} \in \mathrm{D}$ such that $x \notin e_{\alpha_{0}}$. By the compactness of $e_{\alpha_{0}}$, there is a neighborhood $\mathrm{O}(\mathrm{x})$ of $x$ such that $O(x) \cap e_{\alpha_{0}} \neq \emptyset$, and then $O(x) \cap e_{\alpha}=\emptyset$ whenever $\alpha \geqslant \alpha_{0}$. This is a contradiction with $x \in \lim \sup e_{\alpha}$, and hence $\lim e_{\alpha}=e$.

Moreover, Lemma 2.2 implies $\left\{e_{\alpha}\right\}$ converges to e with respect to Hausdorff metric. Hence, for any $0<\beta<\frac{\varepsilon}{2}$, there is $\alpha_{1} \in \mathrm{D}$ such that $e_{\alpha} \subset e+B_{\beta}(0)$ for all $\alpha \geqslant \alpha_{1}$. Since $e_{\alpha}$ is a strongly essential set 
of fix $(T)$, there exists some $\delta>0$ such that $\left[e_{\alpha_{1}}+B_{\beta}(0)\right] \cap \operatorname{fix}\left(T^{\prime}\right) \neq \emptyset$ for any $T^{\prime} \in \operatorname{SP}(T, \delta)$, and then $\left[e+B_{\varepsilon}(0)\right] \cap \operatorname{fix}\left(T^{\prime}\right) \supset\left[e+B_{\beta}(0)+B_{\beta}(0)\right] \cap \operatorname{fix}\left(T^{\prime}\right) \supset\left[e_{\alpha}+B_{\beta}(0)\right] \cap \operatorname{fix}\left(T^{\prime}\right) \neq \emptyset$. By definition of strong essential set, it follows that $e$ is a strongly essential set of $\operatorname{fix}(T)$, i.e. $e \in \mathfrak{R}$. It can be seen that $\mathfrak{R}$ has a minimal element and it is a minimal essential set of fix $(T)$ by means of the standard application of Zorn lemma.

Theorem 3.10. Let $\mathrm{T} \in \mathcal{C}(\mathrm{X})$. Then fix $(\mathrm{T})$ has at least one stable set.

Proof. Suppose that $m(T)$ is a minimal essential set of fix $(T)$. It is sufficient to show that $m(T)$ is connected. If not, then there are nonempty closed subsets $c_{1}$ and $c_{2}$ of $\operatorname{fix}(T)$ such that $m(T)=c_{1} \cup c_{2}$, and since $X$ is compact, there is some $\eta>0$ such that $\left(c_{1}+B_{\eta}(0)\right) \cup\left(c_{2}+B_{\eta}(0)\right)=\emptyset$.

Since $m(T)$ is the minimal element of the family of strongly essential sets of fix $(T), c_{1}$ and $c_{2}$ are not strongly essential. Then for given $\eta$ and any $\delta>0$, there are $T_{1}, T_{2} \in \operatorname{SP}(T, \delta)$ such that

$$
\left(c_{1}+B_{\eta}(0)\right) \cap \operatorname{fix}\left(T_{1}\right)=\emptyset, \quad\left(c_{2}+B_{\eta}(0)\right) \cap \operatorname{fix}\left(T_{2}\right)=\emptyset .
$$

Let $D_{1}=c_{1}+\overline{B_{\frac{\eta}{2}}(0)}$ and $D_{2}=c_{2}+\overline{B_{\frac{\eta}{2}}(0)}$. A partition of unity $\left\{\beta_{1}, \beta_{2}\right\}$ is defined by

$$
\beta_{1}(x)=\frac{d\left(x, D_{2}\right)}{d\left(x, D_{1}\right)+d\left(x, D_{2}\right)}, \quad \beta_{2}(x)=\frac{d\left(x, D_{1}\right)}{d\left(x, D_{1}\right)+d\left(x, D_{2}\right)}, \quad \forall x \in X .
$$

Define a set-valued mapping $T^{\prime}: X \mapsto 2^{X}$ as

$$
T^{\prime}(x)=\beta_{1}(x) T_{1}(x)+\beta_{2}(x) T_{2}(x), \quad \forall x \in X .
$$

Then $T^{\prime}$ is a mapping with nonempty closed and convex value by the definition of $T^{\prime}$.

To prove $T^{\prime} \in \mathcal{C}(X)$, we first show that $T$ is upper semi-continuous. By the compactness of $X$, it is sufficient to prove that the graph of $T^{\prime}$ is closed. For any $x^{n} \rightarrow x, y^{n} \in T^{\prime}\left(x^{n}\right)$ and $y^{n} \rightarrow y$, we should prove that $y \in T^{\prime}(x)$. By the definition of $T^{\prime}$, we have

$$
y^{n} \in T^{\prime}\left(x^{n}\right)=\beta_{1}\left(x^{n}\right) T_{1}\left(x^{n}\right)+\beta_{2}\left(x^{n}\right) T_{2}\left(x^{n}\right)
$$

and hence there are $u^{n} \in T_{1}\left(x^{n}\right)$ and $v^{n} \in T_{2}\left(x^{n}\right)$ such that

$$
y^{n}=\beta_{1}\left(x^{n}\right) u^{n}+\beta_{2}\left(x^{n}\right) v^{n} .
$$

Without loss of generality, assume $u^{n} \rightarrow u$ and $v^{n} \rightarrow v$. Since $T_{1}$ and $T_{2}$ are upper semi-continuous and closed-valued, $u \in T_{1}(x)$ and $v \in T_{2}(x)$. Notice the continuity of $\beta_{1}$ and $\beta_{2}$ and let $n \rightarrow \infty$ in formula (3.2), we have

$$
y=\beta_{1}(x) u+\beta_{2}(x) v \in \beta_{1}(x) T_{1}(x)+\beta_{2}(x) T_{2}(x)=T^{\prime}(x), \quad \forall x \in X
$$

and therefore $T^{\prime}$ is upper semi-continuous. Moreover, $T_{1}, T_{2} \in S P(T, \delta)$ implies

$$
\begin{array}{ll}
\mathrm{T}_{1}(\mathrm{x}) \subset \operatorname{co}\left(\mathrm{T}\left(\mathrm{x}+\mathrm{B}_{\delta}(0)\right)+\mathrm{B}_{\delta}(0)\right), & \forall x \in \mathrm{X}, \\
\mathrm{T}_{2}(\mathrm{x}) \subset \operatorname{co}\left(\mathrm{T}\left(\mathrm{x}+\mathrm{B}_{\delta}(0)\right)+\mathrm{B}_{\delta}(0)\right), & \forall x \in \mathrm{X} .
\end{array}
$$

Then

$$
\begin{aligned}
\mathrm{T}^{\prime}(x) & =\beta_{1}(x) \mathrm{T}_{1}(x)+\beta_{2}(x) \mathrm{T}_{2}(x) \\
& \subset \beta_{1}(x) \operatorname{co}\left(\mathrm{T}\left(x+\mathrm{B}_{\delta}(0)\right)+\mathrm{B}_{\delta}(0)\right)+\beta_{2}(x) \operatorname{co}\left(\mathrm{T}\left(x+\mathrm{B}_{\delta}(0)\right)+\mathrm{B}_{\delta}(0)\right) \subset \operatorname{co}\left(\mathrm{T}\left(x+\mathrm{B}_{\delta}(0)\right)+\mathrm{B}_{\delta}(0)\right),
\end{aligned}
$$

that is, $T^{\prime} \in \operatorname{SP}(T, \delta)$.

However, it is easy to check that $T^{\prime}$ has no fixed points in $D_{1} \cup D_{2}$ by the definition of $T^{\prime}$, and then

$$
\begin{aligned}
& \left(c_{1}+B_{\frac{\eta}{2}}(0)\right) \cap \operatorname{fix}\left(T^{\prime}\right) \subset\left(D_{1}+B_{\frac{\eta}{2}}(0)\right) \cap \operatorname{fix}\left(T^{\prime}\right)=\emptyset, \\
& \left(c_{2}+B_{\frac{\eta}{2}}(0)\right) \cap \operatorname{fix}\left(T^{\prime}\right) \subset\left(D_{2}+B_{\frac{\eta}{2}}(0)\right) \cap \operatorname{fix}\left(T^{\prime}\right)=\emptyset .
\end{aligned}
$$

Notice the arbitrariness of $\delta$ and the definition of strongly essential set. It follows that $m(T)$ is not a strongly essential set of fix $(T)$, a contradiction, and hence $m(T)$ is connected. 
The following corollary is immediate from the connectedness and stable set.

Corollary 3.11. Let $\mathrm{T} \in \mathcal{C}(\mathrm{X})$. Then $\mathrm{fix}(\mathrm{T})$ has at least one strongly essential component.

Proof. Let $m(T)$ be a stable set of fix $(T)$. Since $m(T)$ is connected, it must be in some component $C$ of $\operatorname{fix}(\mathrm{T})$ and then $\mathrm{C}$ is a strongly essential component.

\section{SBR-stable set and stable strategy set of Nash equilibrium points}

Now consider the first problem proposed in Section 1. When the perturbation of strategy sets is sufficiently small, can the perturbation of the best reply correspondence be sufficiently small as well?

Lemma 4.1. Let $\Gamma(\mathrm{X}, \mathrm{f}) \in \Gamma$ and $\mathrm{x} \in \mathrm{X}$. Then for $\varepsilon>0$, there is some $\delta>0$ such that $\mathrm{BR}_{\left(\mathrm{X}^{\prime}, \mathrm{f}\right)}(\mathrm{x}) \subset \mathrm{BR}_{(\mathrm{X}, \mathrm{f})}(\mathrm{x})+$ $\mathrm{B}_{\mathcal{\varepsilon}}(0)$ for all $\Gamma\left(X \mid X^{\prime}, f\right) \in \mathrm{P}(\Gamma(X, f), \delta)$.

Proof. Suppose that it is false. Then there are $\chi^{0}=\left(\chi_{\mathfrak{i}}^{0}, \chi_{-i}^{0}\right) \in X$ and $\varepsilon_{0}>0$, for any $\delta>0$ there are $X^{\delta}=\prod_{i \in N} X_{i}^{\delta}$ and $\Gamma\left(X \mid X^{\delta}, f\right) \in P(\Gamma(X, f), \delta)$ such that $B_{\left(X^{\delta}, f\right)}\left(x^{0}\right) \not \subset \operatorname{BR}_{(X, f)}\left(\chi^{0}\right)+B_{\varepsilon_{0}}(0)$. For each $i \in N$,

$$
\begin{aligned}
\operatorname{BR}_{(X, f)}^{i}\left(x_{-i}^{0}\right) & =\left\{x_{i} \in X_{i}: f_{i}\left(x_{i}, x_{-i}^{0}\right)=\max _{y_{i} \in X_{i}} f_{i}\left(y_{i}, x_{-i}^{0}\right)\right\}, \\
\operatorname{BR}_{\left(X^{\delta}, f\right)}^{i}\left(x_{-i}^{0}\right) & =\left\{x_{i} \in X_{i}^{\delta}: f_{i}\left(x_{i}, x_{-i}^{0}\right)=\max _{y_{i} \in X_{i}^{\delta}} f_{i}\left(y_{i}, x_{-i}^{0}\right)\right\}, \quad \forall i \in N .
\end{aligned}
$$

Let $\max _{x_{i} \in X_{i}} f_{i}\left(x_{i}, x_{-i}^{0}\right)=r_{0}^{i}, X_{i}^{\varepsilon_{0}}=\left\{y_{i} \in X_{i}: d\left(y_{i}, B R_{(X, f)}^{i}\left(x_{-i}^{0}\right)\right) \geqslant \varepsilon_{0}\right\}$, and $\max _{x_{i} \in X^{\varepsilon_{0}}} f_{i}\left(x_{i}, x_{-i}^{0}\right)=q_{0}^{i}$. The definition of $X_{i}^{\varepsilon_{0}}$ implies $x_{i} \notin B R_{(X, f)}^{i}\left(x_{-i}\right)$ for all $x_{i} \in X_{i}^{\varepsilon_{0}}$ and then $f_{i}\left(x_{i}, x_{-i}^{0}\right)<r_{0}^{i}$ and $r_{0}^{i}>q_{0}^{i}$. Let $\eta_{i}=r_{0}^{i}-q_{0}^{i}>0$ and then $\eta_{i}>0$ for each $i \in N$.

Choose some $z^{0}=\left(z_{i}^{0}, z_{-i}^{0}\right) \in B_{(X, f)}\left(x^{0}\right)$. Since $f_{i}$ is continuous on $X$, there is some $\delta^{\prime}>0$ such that for each $i \in N,\left|f_{i}\left(x_{i}^{\prime}, x_{-i}^{0}\right)-f_{i}\left(z_{i}^{0}, x_{-i}^{0}\right)\right|<\frac{\eta_{i}}{3}$ whenever $\left\|x_{i}^{\prime}-z_{i}^{0}\right\|<\delta^{\prime}$, and then

$$
f_{i}\left(x_{i}^{\prime}, x_{-i}^{0}\right)>f_{i}\left(z_{i}^{0}, x_{-i}^{0}\right)-\frac{\eta_{i}}{3}=r_{0}^{i}-\frac{\eta_{i}}{3}=q_{0}^{i}+\eta_{i}-\frac{\eta_{i}}{3}=q_{0}^{i}+\frac{2 \eta_{i}}{3} .
$$

Notice that $\mathrm{BR}_{\left(X^{\delta^{\prime}}, f\right)}\left(x^{0}\right) \not \subset \mathrm{BR}_{(X, f)}\left(x^{0}\right)+\mathrm{B}_{\varepsilon_{0}}(0)$ and choose some $y^{0} \in \mathrm{BR}_{\left(X^{\delta^{\prime}}, \mathrm{f}\right)}\left(\chi^{0}\right)$ such that $y^{0} \notin$ $B R_{(X, f)}\left(x^{0}\right)+B_{\varepsilon_{0}}(0)$. Then for some $i_{0} \in N, y_{i_{0}}^{0} \notin B R_{(X, f)}^{i_{0}}\left(\chi_{-i_{0}}^{0}\right)+B \varepsilon_{\varepsilon_{0}}(0)$, and therefore $y_{i_{0}}^{0} \in X_{i_{0}}^{\varepsilon_{0}}$ and $f_{i_{0}}\left(y_{i_{0}}^{0}, x_{-i_{0}}^{0}\right) \leqslant q_{0}^{i_{0}}$. Moreover, (4.1) implies

$$
f_{i}\left(x_{i}^{\prime}, x_{-i}^{0}\right)>q_{0}^{i}+\frac{2 \eta_{i}}{3}, \quad \forall i \in N,\left\|x_{i}^{\prime}-z_{i}^{0}\right\|<\delta^{\prime} .
$$

Since $\rho\left(X^{\delta^{\prime}}, X\right)<\delta^{\prime}$, there exists some $x_{\mathfrak{i}_{0}}^{\prime} \in X_{\mathfrak{i}_{0}}^{\delta^{\prime}}$ such that

$$
\left\|x_{\mathfrak{i}_{0}}^{\prime}-z_{\mathfrak{i}_{0}}^{0}\right\|<\delta^{\prime},
$$

and then

$$
f_{\mathfrak{i}_{0}}\left(x_{i_{0}}^{\prime}, x_{-i_{0}}^{0}\right)>q_{0}^{i_{0}}+\frac{2 \eta_{i}}{3}>f_{i_{0}}\left(y_{i_{0}}^{0}, x_{-i_{0}}^{0}\right),
$$

which is a contradiction with $y_{i_{0}}^{0} \in \operatorname{BR}_{\left(X^{\delta^{\prime}}, f\right)}^{i}\left(x_{-i}^{0}\right)$.

The following result can be obtained from Lemma 3.6 and Lemma 4.1. 
Lemma 4.2. Let $\Gamma(X, f) \in \Gamma$. For any $\varepsilon>0$, if there is some $\delta>0$ such that

$$
\operatorname{Gr}\left(B_{\left(X^{\prime}, f\right)}\right) \subset \operatorname{Gr}\left(B_{(X, f)}\right)+B_{\varepsilon}(0), \quad \forall \Gamma\left(X \mid X^{\prime}, f\right) \in P(\Gamma(X, f), \delta),
$$

then $\operatorname{BR}_{\left(X^{\prime}, f\right)} \in \operatorname{SP}\left(\mathrm{BR}_{(X, f)}, \varepsilon\right)$.

Proof. Let us prove (4.2). If not, then for given $\varepsilon_{0}>0$ and any $\delta>0$, there exists some $\Gamma\left(X \mid X^{\delta}, f\right) \in$ $P(\Gamma(X, f), \delta)$ such that

$$
\operatorname{Gr}\left(\mathrm{BR}_{\left(X^{\delta}, \mathrm{f}\right)}\right) \not \subset \operatorname{Gr}\left(\mathrm{BR}_{(X, \mathrm{f})}\right)+\mathrm{B}_{\varepsilon_{0}}(0) .
$$

Then there is a sequence of $\left\{\delta_{m}\right\}\left(\delta_{m}>0, \delta_{m} \rightarrow 0\right)$ and the corresponding $X^{m} \in P\left(X, \delta_{m}\right)$ such that

$$
\operatorname{Gr}\left(\operatorname{BR}_{\left(X^{m}, f\right)}\right) \subset \operatorname{Gr}\left(B_{(x, f)}\right)+B_{\varepsilon_{0}}(0)
$$

and hence there is $\left(x^{m}, y^{m}\right) \in \operatorname{Gr}\left(B_{\left(X^{m}, f\right)}\right)$ such that $\left(x^{m}, y^{m}\right) \notin \operatorname{Gr}\left(B R_{(X, f)}\right)+B_{\varepsilon_{0}}(0)$. Since $X$ is compact, without loss of generality, assume $\left(x^{m}, y^{m}\right) \rightarrow(x, y)$, and then $(x, y) \notin \operatorname{Gr}\left(B R_{(X, f)}\right)+B_{\varepsilon_{0}}(0)$, i.e. $y \notin$ $B R_{(X, f)}(x)+B_{\varepsilon_{0}}(0)$. Therefore, there is some $i \in N$ such that $y_{i} \notin B R_{(X, f)}^{i}\left(x_{-i}\right)+B_{\varepsilon_{0}}(0)$, i.e. there exists some $x_{i}^{\prime} \in X_{i}$ such that $f_{i}\left(y_{i}, x_{-i}\right)<f_{i}\left(x_{i}^{\prime}, x_{-i}\right)$. Let $f_{i}\left(x_{i}^{\prime}, x_{-i}\right)-f_{i}\left(y_{i}, x_{-i}\right)=\eta>0$. Since $X^{m} \in P\left(X, \delta_{m}\right)$, we have $H_{d}\left(X_{i}^{m}, X_{i}\right)<\delta_{m}$ for each $i \in N$, where $H_{d}$ is the Hausdorff distance between $X_{i}^{m}$ and $X_{i}$. Since $x_{i}^{\prime} \in X_{i}$ and $H_{d}\left(X_{i}^{m}, X_{i}\right)<\delta_{m}$, there is some $z_{i}^{m} \in X_{i}^{m}$ such that $\left\|z_{i}^{m}-x_{i}^{\prime}\right\|<\delta_{m}$ and then $z_{i}^{m} \rightarrow x_{i}^{\prime}$. Notice that $X$ is compact and $f_{i}$ is continuous on $X$. We have the uniform continuity of $f_{i}$ on $X$. Consider $f_{i}\left(x_{i}^{\prime}, x_{-i}\right)-f_{i}\left(y_{i}, x_{-i}\right)=\eta$ and $z_{i}^{m} \rightarrow x_{i}^{\prime}, x^{m} \rightarrow x, y^{m} \rightarrow y$. There is some $N_{0}>0$ such that for all $m>N_{0}$, we have

$$
\left|f_{i}\left(z_{i}^{m}, x_{-i}^{m}\right)-f_{i}\left(x_{i}^{\prime}, x_{-i}^{m}\right)\right|<\frac{\eta}{3}, \quad\left|f_{i}\left(x_{i}^{\prime}, x_{-i}^{m}\right)-f_{i}\left(x_{i}^{\prime}, x_{-i}\right)\right|<\frac{\eta}{3}, \quad\left|f_{i}\left(y_{i}, x_{-i}\right)-f_{i}\left(y_{i}^{m}, x_{-i}^{m}\right)\right|<\frac{\eta}{3} .
$$

Furthermore,

$$
\begin{aligned}
f_{i}\left(z_{i}^{m}, x_{-i}^{m}\right)-f_{i}\left(y_{i}^{m}, x_{-i}^{m}\right)= & f_{i}\left(z_{i}^{m}, x_{-i}^{m}\right)-f_{i}\left(x_{i}^{\prime}, x_{-i}^{m}\right)+f_{i}\left(x_{i}^{\prime}, x_{-i}^{m}\right)-f_{i}\left(x_{i}^{\prime}, x_{-i}\right) \\
& +f_{i}\left(x_{i}^{\prime}, x_{-i}\right)-f_{i}\left(y_{i}, x_{-i}\right)+f_{i}\left(y_{i}, x_{-i}\right)-f_{i}\left(y_{i}^{m}, x_{-i}^{m}\right) \\
> & -\frac{\eta}{3}-\frac{\eta}{3}+\eta-\frac{\eta}{3}=0 .
\end{aligned}
$$

Therefore, $f_{i}\left(z_{i}^{m}, x_{-i}^{m}\right)>f_{i}\left(y_{i}^{m}, x_{-i}^{m}\right)$, and hence $y_{i}^{m} \notin B R_{\left(X^{m}, f\right)}^{i}\left(x_{-i}^{m}\right)$, i.e. $y^{m} \notin B R_{\left(X^{m}, f\right)}\left(x^{m}\right)$. This is a contradiction. Then it is immediate from Lemma 3.6 and $(4.2)$ that $\operatorname{BR}_{\left(X^{\prime}, f\right)} \in \operatorname{SP}\left(B R_{(X, f)}, \varepsilon\right)$.

The following conclusions is from Lemma 4.1 and Lemma 4.2.

(I) For each fixed point, the perturbation of values of the best reply correspondence is sufficiently small whenever the perturbation of strategy sets is sufficiently small.

(II) The strong $\delta$-perturbation of the best reply correspondence is sufficiently small whenever the perturbation of strategy sets is sufficiently small.

Lemma 3.6 shows that the class of perturbed games considered in the definition of strong $\delta$-perturbation is richer than those considered in the definitions of essential sets considered by McLennan [13], especially BR-stability. Lemma 4.1 shows that SBR-stable set can be used to give the stability to perturbations of strategy sets. So the strong $\delta$-perturbation of the best reply correspondence will be used to define an appropriate stable set for Nash equilibria, called SBR-stable set. Theorem 4.5 below will show the existence and connectedness of SBR-stable and answer the second problem proposed in Section 2.

Definition 4.3. Let $\Gamma(X, f) \in \Gamma, B R_{(X, f)}$ the best reply correspondence of game $\Gamma(X, f)$, and $S \subset E(X, f)$ a closed set. If for any $\varepsilon>0$, there is some $\delta>0$ such that $\left(S+B_{\varepsilon}(0)\right) \cap \operatorname{fix}\left(T^{\prime}\right) \neq \emptyset$ for all $T^{\prime} \in \operatorname{SP}\left(B R_{(X, f)}, \delta\right)$, then $S$ is said to be an $S B R$-set of $E(X, f)$. If $S$ is a minimal element of the family of all the $S B R$-sets of $E(X, f)$ with partial order defined by the inclusion relation, then $S$ is said to be an $S B R$-stable set of $E(X, f)$. 
Remark 4.4. In Definition 4.3, if the $S B R$-set is defined by replacing strong $\delta$-perturbation $\operatorname{SP}\left(B R_{(X, f)}, \delta\right)$ with $\delta$-perturbation $P\left(B R_{(X, f)}, \delta\right)$ and $\operatorname{GP}\left(B R_{(X, f)}, \delta\right)$, respectively, then the SBR-set becomes a BR-set or an essential set.

For game $\Gamma(X, f) \in \Gamma$ and its best reply correspondence $B R_{(X, f)}$ (BR for short), a strong $\delta$-perturbation is expressed as

$$
\mathrm{SP}(\mathrm{BR}, \delta)=\left\{\mathrm{S} \in \mathcal{C}(\mathrm{X}): \mathrm{S}(\mathrm{x}) \subset \operatorname{co}\left(\mathrm{BR}\left(\mathrm{x}+\mathrm{B}_{\delta}(0)\right)+\mathrm{B}_{\delta}(0)\right), \forall x \in X\right\} .
$$

Let us explain briefly the meaning of strong $\delta$-perturbation in some way. A strong $\delta$-perturbation is to be interpreted as modelling mistakes made by the players with some negligible probability when the game is played. Such mistakes would most likely consist of a player making another move than the one intended at some point during play. Some understanding is as follows.

(A) " $\mathrm{B}_{\delta}(0)$ " in "BR $\left(x+\mathrm{B}_{\delta}(0)\right)^{\prime \prime}$ can be explained as the mistakes that players sometimes aim at a different strategy profile than $x$ when they intend to select the best response to strategy profile $x$ (such as some $\left.x^{\prime} \in x+B_{\delta}(0)\right)$.

(B) The second " $\mathrm{B}_{\delta}(0)$ " in "BR( $\left.\mathrm{B}+\mathrm{B}_{\delta}(0)\right)+\mathrm{B}_{\delta}(0)$ " can be explained as the mistakes that players sometimes make another move than some $y \in B R\left(x+B_{\delta}(0)\right)$ when they intend to play the best reply to strategy profile $x^{\prime} \in x+B_{\delta}(0)$ (for example, some $y^{\prime} \in B R\left(x+B_{\delta}(0)\right)+B_{\delta}(0)$ ).

(C) The convex hull "co" in " $\mathrm{co}\left(\mathrm{BR}\left(x+\mathrm{B}_{\delta}(0)\right)+\mathrm{B}_{\delta}(0)\right)$ " can be explained as the possibility that players make the mistakes combined by perturbations generated in the cases $(A)$ and $(B)$ when they intend to play the best reply to strategy profile $x$. Here the perturbations are similar to the mixed strategies.

Theorem 4.5. Let $\Gamma(X, f) \in \Gamma$. Then $\mathrm{E}(\mathrm{X}, \mathrm{f})$ has at least one SBR-stable set.

Proof. Suppose BR is the best reply correspondence of game $\Gamma(X, f)$. By Theorem 3.10, fix(BR) has a stable set $S \subset \operatorname{fix}(B R)$. Then for any $\varepsilon>0$, there is some $\delta>0$ such that $\left(S+B_{\varepsilon}(0)\right) \cap \operatorname{fix}(T) \neq \emptyset$ for all $T \in \operatorname{SP}(B R, \delta)$. Notice $E(X, f)=\operatorname{fix}(B R)$. We have $S \subset E(X, f)$ and $\left(S+B_{\varepsilon}(0)\right) \cap E(X, f) \neq \emptyset$, and then $S$ is an $S B R$-stable set of $E(X, f)$ by Definition 4.3.

Finally, it will be proved that an SBR-stable set is stable to any perturbations of strategy sets, so that it has the strategic stability.

Definition 4.6. Let $\Gamma(X, f) \in \Gamma$, and $S \subset E(X, f)$ be a nonempty closed subset. For any $\varepsilon>0$, if there is $\delta>0$ such that $\left(S+B_{\varepsilon}(0)\right) \cap E\left(X^{\prime}, f\right) \neq \emptyset$ for all $\Gamma\left(X \mid X^{\prime}, f\right) \in P(\Gamma(X, f), \delta)$ and corresponding game $\Gamma\left(X^{\prime}, f\right)$, then $S$ is said to be a strategically essential set.

Theorem 4.7. Let $\Gamma(X, f) \in \Gamma$, and $S \subset E(X, f)$ be an $S B R$-stable set of $E(X, f)$. Then $S$ is a strategically essential set.

Proof. By Theorem 4.5, $\mathrm{E}(\mathrm{X}, \mathrm{f})$ has at least one SBR-stable set, denoted as $\mathrm{S}$. It only needs to prove that $S$ is a strategically essential set. Since $S$ is an $S B R$-stable set, for any $\varepsilon>0$, there is some $\eta>0$ such that $\left(S+B_{\varepsilon}(0)\right) \cap \operatorname{fix}(T) \neq \emptyset$ for all $T \in S P\left(B R_{(X, f)}, \eta\right)$. By Lemma 4.2, for given $\eta>0$, there is some

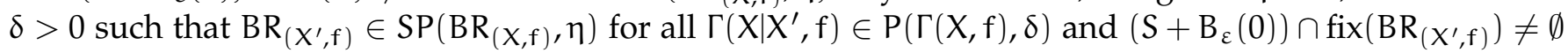
for all $\Gamma\left(X \mid X^{\prime}, f\right) \in P(\Gamma(X, f), \delta)$. Notice fix $\left(B R_{\left.\left(X^{\prime}, f\right)\right)}\right)=E\left(X^{\prime}, f\right)$. Then $\left(S+B_{\varepsilon}(0)\right) \cap E\left(X^{\prime}, f\right) \neq \emptyset$ for all $\Gamma\left(X \mid X^{\prime}, f\right) \in P(\Gamma(X, f), \delta)$, and hence $S$ is a strategically essential set.

Theorems 4.5 and 4.7 show that each $\Gamma(X, f) \in \Gamma$ has at least one strategically essential set.

Since an SBR-stable set has stability with respect to any strong $\delta$-perturbation and various perturbations for a strategy set are not beyond the range of a strong $\delta$-perturbation, an SBR-stable set has the stability that various essential sets possess, such as fully-stable set, stable set, quasistable set and essential set. An SBR-stable set is stable to any perturbation of strategy sets defined directly by Hausdorff distance without any additional conditions, so the concept of SBR-stable set will provide convenience for studying the strategic stability, which is even used to study any noncooperative finite game. 


\section{Acknowledgment}

This work is supported by the National Natural Science Foundation of P. R. China (No.70661001, 11161008, 11561013) and the Doctoral Program Foundation of Ministry of Education of P. R. China (No.20115201110002).

\section{References}

[1] O. Carbonell-Nicolau, On strategic stability in discontinuous games, Econom. Lett., 113 (2011), 120-123. 1

[2] O. Carbonell-Nicolau, Further results on essential Nash equilibria in normal-form games, Econom. Theory, 59 (2015), 277-300. 1

[3] M. K. Fort, Jr., Points of continuity of semi-continuous functions, Publ. Math. Debrecen, 2 (1951), 100-102. 2

[4] S. Govindan, R. Wilson, Essential equilibria, Proc. Natl. Acad. Sci. USA, 102 (2005), 15706-15711. 1

[5] J. Hillas, On the definition of the strategic stability of equilibria, Econometrica, 58 (1990), 1365-1390. 1

[6] J. Hillas, M. Jansen, J. Potters, D. Vermeulen, On the relation among some definitions of strategic stability, Math. Oper. Res., 26 (2002), 611-635. 1

[7] J. Hillas, M. Jansen, J. Potters, D. Vermeulen, Independence of inadmissible strategies and best reply stability: a direct proof, Special issue on stable equilibria, Internat. J. Game Theory, 32 (2004), 371-377.

[8] W.-S. Jia, S.-W. Xiang, J.-H. He, Y.-L. Yang, Existence and stability of weakly Pareto-Nash equilibrium for generalized multiobjective multi-leaderfollower games, J. Global Optim., 61 (2015), 397-405. 1

[9] E. Kalai, D. Samet, Persistent equilibria in strategic games, Internat. J. Game Theory, 13 (1984), 129-144. 1

[10] E. Klein, A. C. Thompson, Theory of correspondences, Including applications to mathematical economics, Canadian Mathematical Society Series of Monographs and Advanced Texts, A Wiley-Interscience Publication, John Wiley \& Sons, Inc., New York, (1984). 2

[11] E. Kohlberg, J. F. Mertens, On the strategic stability of equilibria, Econometrica, 54 (1986), 1003-1037. 1, 3

[12] D. M. Kreps, R. Wilson, Sequential equilibria, Econometrica, 50 (1982), 863-894. 1

[13] A. McLennan, Fixed points of contractible valued correspondences, Internat. J. Game Theory, 18 (1989), 175-184. 1, 3, 4,4

[14] J. F. Mertens, Stable equilibria-a reformulation, I, Definition and basic properties, Math. Oper. Res., 14 (1989), $575-$ 625. 1

[15] R. B. Myerson, Refinements of the Nash equilibrium concept, Internat. J. Game Theory, 7 (1978), 73-80. 1

[16] A. B. Sadanand, V. Sadanand, Equilibria in non-cooperative games, I, Perturbations based refinements of Nash equilibrium, Bull. Econ. Res., 43 (1994), 197-224. 1

[17] V. Scalzo, Essential equilibria of discontinuous games, Econom. Theory, 54 (2013), 27-44. 1

[18] V. Scalzo, On the existence of essential and trembling-hand perfect equilibria in discontinuous games, Econ. Theory Bull., 1 (2013), 1-12. 1

[19] R. Selten, Reexamination of the perfectness concept for equilibrium points in extensive games, Internat. J. Game Theory, 4 (1975), 25-55. 1, 3

[20] E. van Damme, Strategic equilibrium, Handbook of Game Theory with Economic Applications, 3 (2002), 1521-1596. 1

[21] A. J. Vermeulen, J. A. M. Potters, M. J. M. Jansen, On quasi-stable sets, Internat. J. Game Theory, 25 (1996), $43-49$.

[22] A. J. Vermeulen, J. A. M. Potter, M. J. M. Jansen, On stable sets of equilibria, Game theoretical applications to economics and operations research, Bangalore, (1996), Theory Decis. Lib. Ser. C Game Theory Math. Program. Oper. Res., Kluwer Acad. Publ., Boston, MA, 18 (1997), 133-148. 1

[23] W.-T. Wu, J.-H. Jiang, Essential equilibrium points of n-person non-cooperative games, Sci. Sinica, 11 (1962), $1307-1322$. 1

[24] S.-W. Xiang, G.-D. Liu, Y.-H. Zhou, On the strongly essential components of Nash equilibria of infinite n-person games with quasiconcave payoffs, Nonlinear Anal., 63 (2005), e2637-e2647. 1

[25] J. Yu, Q. Luo, On essential components of the solution set of generalized games, J. Math. Anal. Appl., 230 (1999), 303-310. 1

[26] J. Yu, S.-W. Xiang, On essential components of the set of Nash equilibrium points, Nonlinear Anal., 38 (1999), $259-264$. 1

[27] Y.-H. Zhou, J. Yu, S.-W. Xiang, Essential stability in games with infinitely many pure strategies, Internat. J. Game Theory, 35 (2007), 493-503. 1 